\title{
An exposure guided background subtraction for smart camera
}

\author{
Tianyi Chen ${ }^{1,2}$, Xiaoxu Zheng ${ }^{1,2}$, Bin Wang ${ }^{1,2, a,{ }^{*}}$, Wen Gai $^{1,2}$, Maojun Zhang ${ }^{3}$ \\ ${ }^{1}$ Facility Design and Instrumentation Institute, China Aerodynamics Research and Development \\ Center, Mianyang 621000, China \\ ${ }^{2}$ State Key Laboratory of Aerodynamics, China Aerodynamics Research and Development Center, \\ Mianyang 621000, China \\ ${ }^{3}$ College of Information System and Management, National University of Defense Technology, \\ Changsha 410073, China \\ anudtwangbin@163.com
}

Keywords: smart camera, background subtraction, auto-exposure, visual surveillance.

\begin{abstract}
An exposure guided background subtraction (EGBS) model is proposed for smart cameras to handle illumination change due to auto-exposure in visual surveillance. To reduce false foreground pixels caused by auto-exposure, EGBS compensates background illumination directly utilizing the information generated by auto-exposure module without extra illumination change estimation. Hence, it is very preferable for smart camera without any extra hardware resources. Experimental results indicate the proposed model efficiently reduces false foreground pixels caused by auto-exposure.
\end{abstract}

\section{Introduction}

Background subtraction is one of the most widely used techniques to segment moving objects for static cameras in visual surveillance system. Many useful background subtraction methods such as mixture of Gaussian (MoG) ${ }^{[1]}$, codebook and Vibe ${ }^{[2]}$ have been proposed. However, most of them can't cope with fast illumination change ${ }^{[3-5]}$ caused by light change or auto-exposure. In visual surveillance application, almost every surveillance camera supports auto-exposure to adapt to different illuminations. For example, in indoor visual surveillance, auto-exposure frequently occurs due to object moving while the background scene illumination doesn't change. When auto-exposure occurs, both the fixed background pixels and moving object pixels are all detected as foreground pixels.

In order to handle the fast illumination change, some illumination invariant features such as gradient or edge are used for background modeling. Gradient information is most discriminating at the boundaries of the objects, but does not provide a clear difference for large, untested objects (e.g. a white bus on the road surface). Furthermore, gradient information used for pixel-based background subtraction is highly unreliable when camera moves (such as shaking). Another way to handle illumination change is background illumination compensation, which considers the illumination compensation as an inverse problem. They ${ }^{[6-7]}$ first estimate the illumination change and then compensate the background illumination. However, these algorithms require high estimation accuracy and high computational cost to estimate the illumination change. Therefore, these methods are not suitable for smart cameras because of the limited resources, such as energy, processing power (egg: battery-powered camera) and memory in the cameras ${ }^{[8]}$.

\section{Proposed method}

In this paper, we propose a method that does not need to estimate the illumination change, and we get this information from the source of imaging sensor. Thanks to the advances in silicon manufacturing technology, intelligent analysis and ISP (image signal processor, which integrates an auto-exposure module) can be integrated into a single chip for smart cameras (Fig.1.a). This integration provides a novel way to handle illumination change caused by auto-exposure. We can utilize the information 
generated by auto-exposure model of ISP to guide background illumination compensation (Fig.1.b). We propose an exposure guided background subtraction algorithm based on MoG. It directly uses exposure information to guide the MoG background model precisely updating before background subtraction, reducing the false foreground pixels in background. Benefiting from using the hardware information in camera, the proposed method doesn't need any extra hardware resources to estimate illumination change, so it is suitable for resource limited smart cameras. To the best of our knowledge, this is the first effort exploring utilizing the front-end device information (such as image signal processor, ISP) to improve intelligent analysis performance in smart cameras.

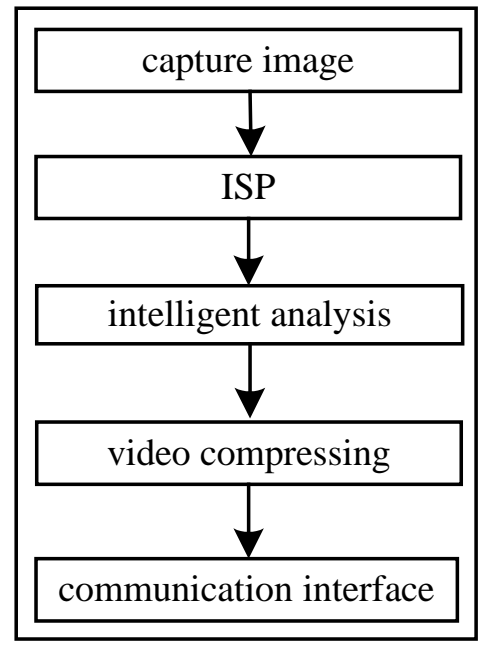

(a)

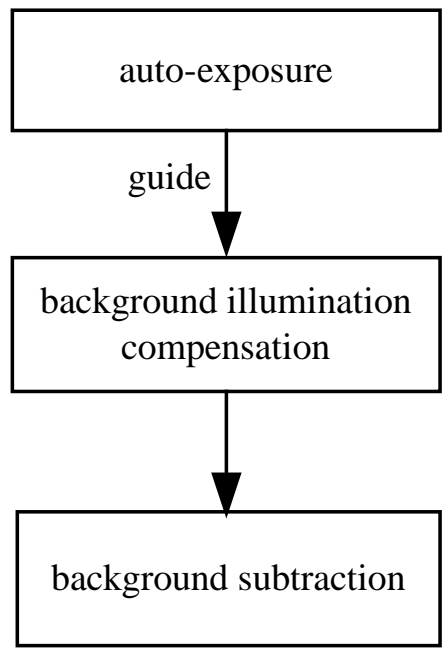

(b)

Fig.1 (a) smart camera, (b) exposure guided background subtraction

\section{Exposure guided MoG background modelling}

In typical video cameras, the auto-exposure module of ISP adjusts image brightness with brightness change ratio $\mathrm{R}$. And $\mathrm{R}$ is calculated as equation (1). The changed background illumination can be precisely compensated by utilizing $\mathrm{R}$.

$R=$ CurBrightness $/ T$ arg etBrightness

Mixture of Gaussians (MoG) is a commonly used background subtraction method in visual surveillance since it can cope with periodic disturbances (swaying vegetation or flowing water). However, it can't cope with fast illumination change caused by auto-exposure. So we choose MoG as the baseline method and handle the fast illumination change caused by auto-exposure by utilizing the exposure information of camera.

The MoG method models background pixel's value distribution using K-Gaussians, and describe the probability of observing a pixel value $X_{t}$ at time $t$ as (2). $\mathrm{K}$ is the number of Gaussians, which is set to be 3 in our experiment. $\omega_{i, t}, u_{i, t}$ and $\Sigma_{i, t}$ are weight, mean and the covariance matrix of itch Gaussian in the mixture at time t, which are learned from a background sequence $B=\left(b_{1}, b_{2} \ldots b_{n}\right)$.

$$
P\left(X_{t}\right)=\sum_{i=1}^{K} \omega_{i, t} \eta\left(X_{t}, u_{i, t}, \Sigma_{i, t}\right)
$$

At time t, when an object moves and changes the average brightness of current frame (in Fig.2.b), auto-exposure of ISP in smart camera will occur. The value of pixel in background ${ }^{X_{t}}$ will be changed to $X_{t}{ }^{\prime}$ with $\mathrm{R}$ as (3). $\mathrm{R}$ is the adjust factor (equation.1).

$$
X_{t}{ }^{\prime}=R X_{t}
$$

Then, $X_{t}{ }^{\prime}$ no longer meets the background distribution which is only supported by original background sequence $B$. It leads to be set to false foreground. If the background sequence $B$ is 
adjusted as (4), $X_{t}^{\prime}$ will meet to the adjusted background distribution which is supported by B'. And $x_{t}^{\prime}$ will avoid to be set to false foreground.

$$
B^{\prime}=\left(b_{1}^{\prime}, b_{2}^{\prime} \ldots b_{n}^{\prime}\right)=R\left(b_{1}, b_{2} \ldots b_{n}\right)
$$

Now, the K-Gaussians background model also needs to be adjusted to fit the new distribution of the adjusted background sequence B'. The K-Gaussians model is linear, in which, every component is a single Gaussian model with mean $u_{i}$ and variance $\sigma_{i}$. After being adjusted as (4), the background pixels mean $u_{i}$ and variance $\sigma_{i}$ are respectively changed to $u_{i}^{\prime}$ and $\sigma_{i}^{\prime}$ as (5) and (6), where $n_{i}$ is the number of pixels belong to itch Gaussian, $b_{j}^{i}$ and $b^{i}{ }_{j}$ are the pixels belong to itch Gaussian before and after auto-exposure adjusting.

$$
\begin{aligned}
& u_{i}^{\prime}=\frac{1}{n_{i}} \sum_{j=1}^{n_{i}} b_{j}^{{ }^{i}}=\frac{1}{n_{i}} \sum_{j=1}^{n_{i}} R b_{j}^{i}=R u_{i} \\
& \sigma_{i}^{\prime}=\sqrt{E\left(b^{i^{i}{ }_{j}}\right)-\left(u_{i}^{\prime}\right)^{2}}=\sqrt{\frac{1}{n_{i}} \sum_{j=1}^{n_{i}}\left(b^{\prime i}{ }_{j}\right)^{2}-\left(u_{i}^{\prime}\right)^{2}}=\sqrt{\frac{1}{n_{i}} \sum_{j=1}^{n_{i}}\left(R b_{j}^{i}\right)^{2}-\left(R u_{i}\right)^{2}}=R \sigma_{i}
\end{aligned}
$$

Since K-Gaussian mixture model is linear, so when auto-exposure occurs, the probability of observing a pixel value ${ }^{X^{\prime}}{ }_{t}$ at time $t$ can be described as (7). ${ }^{\Sigma_{i, t}}$ Is equivalent to ${ }^{\sigma_{i}}$, since we assume that each color channel of RGB is independent?

$$
\begin{aligned}
& P\left(X_{t}^{\prime}\right)=\sum_{i=1}^{K} \omega_{i, t} \eta\left(X_{t}^{\prime}, u_{i, t}^{\prime}, \Sigma_{i, t}^{\prime}\right) \\
& u_{i, t}^{\prime}=R u_{i, t} \\
& \Sigma_{i, t}^{\prime}=R \Sigma_{i, t}=R \sigma_{i, t}
\end{aligned}
$$

The following steps to classify the pixels to foreground or background are like MoG.

\section{Experiment and results}

We designed a smart camera which is an embedded system based on FPGA to evaluate our proposed method EGBS. We capture 10 test videos in indoor surveillance; Fig.2 illustrates one of these video pictures, and the foreground detection results are also shown in Fig.2. The quantization performance indicators of 10 test videos are shown in Table 1. We can conclude that, compared with the MoG method, the proposed method improves segmentation performance with a higher F-score against auto-exposure from the experiments.

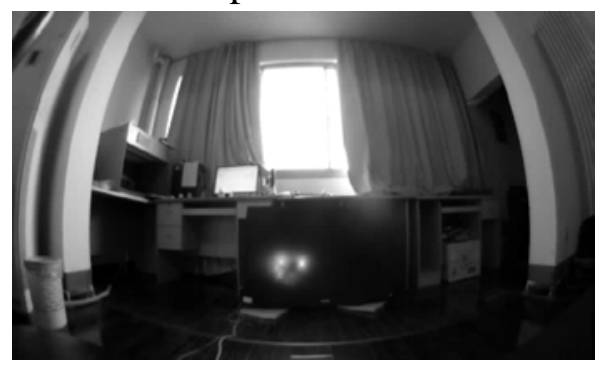

a

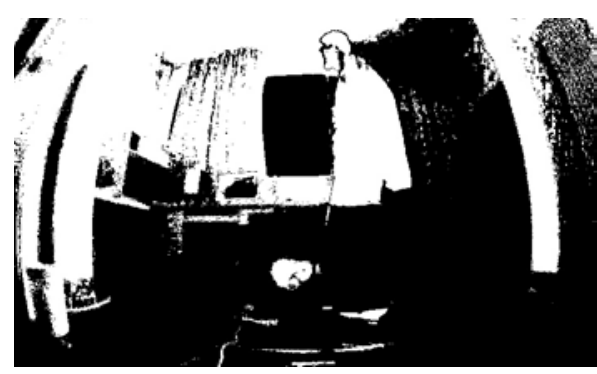

C

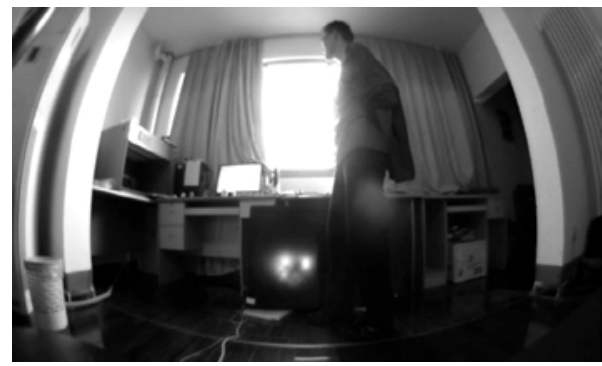

b

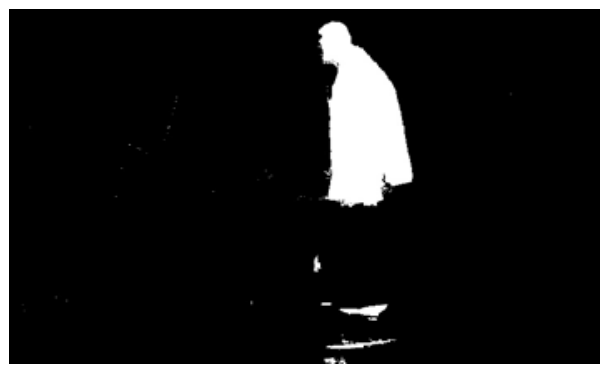

d 
Fig.2 Results of foreground detection in one test video, a: Background image, b: Auto-exposure image, c: MoG, d: Exposure guide MoG

Table 1: Performance indicators of MoG and proposed method

\begin{tabular}{|c|c|c|}
\hline Average (\%) & MoG & EGBS (EGMoG) \\
\hline Precision & 22.31 & 80.15 \\
\hline Recall & 42.22 & 72.24 \\
\hline F-score & 28.94 & 69.56 \\
\hline
\end{tabular}

\section{Discussions}

Directly using the exposure information to guide background model illumination compensation is an effective method to handle illumination change caused by auto-exposure. It's a light-weight and efficient method with less computational cost. And it is suitable for smart cameras. In the future work, we will extensively explore the combination of ISP and intelligent analysis algorithms to enhance the smart camera performance with less resource consumption.

\section{Reference}

[1] Stauffer C, Grimson W E L. Learning patterns of activity using real-time tracking [J]. Pattern Analysis and Machine Intelligence, IEEE Transactions on, 2000, 22(8): p.747-757.

[2] Barnich O, Van Droogenbroeck M. ViBe: A universal background subtraction algorithm for video sequences [J]. Image Processing, IEEE Transactions on, 2011, 20(6): p.1709-1724.

[3] Wang B, Liu Y, Xu W, et al. Background subtraction using spatiotemporal condition information [J]. Optik-International Journal for Light and Electron Optics, 2014, 125(3): p.1406-1411.

[4] Liu L Y, Sang N, Huang R. Background subtraction using shape and colour information[J]. Electronics letters, 2010, 46(1): p.41-43.

[5] Choi J M, Chang H J, Yoo Y J, et al. Robust moving object detection against fast illumination change[J]. Computer Vision and Image Understanding, 2012, 116(2): p.179-193.

[6] Parameswaran V, Singh M, Ramesh V. Illumination compensation based change detection using order consistency[C]//Computer Vision and Pattern Recognition (CVPR), 2010 IEEE Conference on. IEEE, 2010: p.1982-1989.

[7] Moore D J. Dynamic Illumination Compensation for Background Subtraction: U.S. Patent Application 13/190,404[P]. 2011-7-25.

[8] Casares M, Velipasalar S, Pinto A. Light-weight salient foreground detection for embedded smart cameras [J]. Computer Vision and Image Understanding, 2010, 114(11): p.1223-1237. 